\title{
MODELING OF THRESHOLD EFFECTS IN SOCIAL SYSTEMS BASED ON NONLINEAR DYNAMICS
}

\author{
Alexandr Y. Petukhov \\ Research lab. "Modeling of social and political processes" \\ Lobachevsky Nizhny Novgorod State University \\ Russia \\ Lectorr@yandex.ru
}

Article history:

Received 15.10.2019, Accepted 26.11.2019

\begin{abstract}
This study proposes a model of threshold effects in social processes under conflict conditions. A model based on the diffusion equation of Langevin is developed. A solution of the system of equations for a divergent diffusion type is given. Using the example of two interactingconflicting groups of individuals, we have identified the characteristic patterns of social conflict in the social system in terms of threshold effects and determined the effect the social distance in society has in development of similar processes with regard to the external influence, dissipation, and random factors. We have demonstrated how the phase portrait of the system qualitatively changes as the parameters of the control function of the social conflict change in terms of threshold effects. Using the analysis data of the resulting phase portraits, we have concluded about the existence of a characteristic region of sustainability determined by the transition processes in terms of the threshold effect in the social system, within which it is relatively stable.
\end{abstract}

\section{Key words}

Socium, Langevin equation, field of communication, control.

\section{Introduction}

Social conflict is a classic threshold effect for social systems. A social conflict can be defined as a peak stage in the development of contradictions in relations between individuals, groups of individuals, or a society as a whole, characterized by the presence of contradicting interests, objectives and viewpoints of the interacting subjects. Conflicts can be latent or explicit, and are caused by lack of a compromise or sometimes even a dialogue between the two or more parties involved [Petukhov, 2015].

Works of the foreign scientists that became fundamental in analyzing practical problems of this complex interdisciplinary science played an important part in the development of general conflictology at the present stage.

One of the most successful and consistent approaches to the modeling of social phenomena with the help of discrete equations was carried out by D. Hayes [Malkov, 2009].

The problems of studying, classifying, and the most important - predicting conflicts always played a significant role in fundamental social science. A lot of works of leading sociologists and mathematicians were devoted to the studying of this topic [Castellano, 2009], [Smith, 2013], [Traud, 2011].

In recent years, significant progress has been made in the development of models of social and political processes [Abzalilov, 2012].

The models available to date can be divided into three groups:

1) models - concepts based on the identification and analysis of common historical patterns and their representation in the form of cognitive schemes that describe the logical connections between various factors that affect historical processes [Malkov, 2009]. Such models generalize the subject matter to a high degree, but they are not of a mathematical, but of purely logical, conceptual nature;

2) special mathematical models of imitative type, created for the description of specific historical events and phenomena [Malkov, 2009]. Such models focus on careful registration and description of the factors and processes that affect the phenomena under consideration. Applicability of such models, as a rule, is limited by a rather narrow space-time interval; they are "tied" to a 
specific historical event and they cannot be extrapolated for extended periods of time;

3) mathematical models, which are intermediate between the two-abovementioned types. These models describe a certain class of social processes without claiming to provide a detailed description of the features for each particular historical event. Their task is to identify the basic regularities characterizing the course of the processes of the discussed type. In this regard, these mathematical models are called basic models [Malkov, 2009].

Holyst J.A., Kacperski K., Schweiter F. propose a convenient model of public opinion, which views the interaction between individuals as a Brownian motion [Holyst, 2000].

In addition, the problem of modeling social conflicts was investigated in previous works of the author. In this paper, we considered a model without introducing a control function and its effect on conflict processes [Petukhov, 2018].

Taking into account the important impact of such phenomena on a society and all the processes inside it, any ways of predicting and discovering characteristic laws of social conflicts are certainly of a paramount importance.

A separate direction in this area is a look at social conflict as a threshold effect in a complex cognitive system.

\section{Model}

It is important to identify a parameter determinant to a social conflict, which will underlie the model we are creating. It is clear that this parameter should be logically justified within the framework of the main modern concepts of social conflict.

This parameter is social distance. Previous works [Petukhov, 2018] discuss this matter in more detail. Therefore, here we will only provide the following provisions critical for understanding of this model:

1. A major social conflict, as a rule, is accompanied by an informational and social distance between individuals and groups of individuals. Such a distance can be based on interethnic, cultural, religious, and economic differences. There can be various reasons for such a conflict: different levels of aggression of social and ethnic groups, contradicting cultural and economic aspirations, etc. Thus, the social-informational distance itself does not cause the conflict, but, as a rule, accompanies it.

2. This distance increases during the course of the conflict, especially in its extreme variants (revolutions, civil wars, etc.), leading the opposing parties to the position of "non-reconciliation". The history, unfortunately, has very few examples of short and medium-term positive scenario for such situations.

3. Therefore, this point of no return, as a rule, occurs just before the onset of the conflict, and such a transition of a social system from one state to another become decisive (triggering) for the overall situation.

In this case, as a rule, very few conflicts in a modern globalizing world occur without external influence and even interference. This raises the question of introducing control into a model of conflict. This control can play a decisive role in its generation and dynamics.

\subsection{Fundamentals of the Model}

Socio-political processes are subject to constant changes and deformations, therefore from the point of view of mathematical modeling they cannot be set with a high degree of precision. Here we can trace the analogy with the Brownian particle, i.e. a particle that seemingly moves along a rather defined trajectory, but under close examination, this trajectory turns out to be strongly tortuous, with many small knees. These small changes (fluctuations) are explained by the chaotic motion of other molecules. In social processes, fluctuations can be interpreted as manifestations of the free will of its individual participants, as well as other random manifestations of the external environment [Petukhov, 2018].

In physics, these processes are, as a rule, described by Langevin equation of the stochastic diffusion, which has been applied with relative success for modeling of some social processes as well.

This approach has several advantages:

1. As it has already been mentioned, the approach allows taking into account the manifestations of the free will of its individual participants, as well as other random manifestations of the external environment for the social system.

2. The behavior of a social system can be calculated, both for its entirety, and for separate individuals.

3. This approach allows identifying some distinctive stable modes of functioning of social systems, depending on various initial conditions.

4. Diffusion equations, as a mathematical apparatus, have been sufficiently validated and studied from the point of view of numerical simulation.

The model is based on the assumption that individuals interact in society through a communicative field $h$. This field is induced by each individual in society and serves as a model of the information interaction between individuals. However, we should keep in mind that here we are talking about a society, which is difficult to classify as an object in classical physical spatial topology. Objectively, from the point of view of information transfer from an individual to an individual, space in society combines both classical spatial coordinates and additional specific parameters and features. This is caused by the fact that in the modern information world there is no need to be close to the object of influence in order to transmit information to it.

Thus, the society is a multidimensional, socialphysical space that reflects the ability of one individual to "reach" another individual with his communicative field, that is, to influence it, its parameters and the ability to move in a given space. Accordingly, the position of the individual relative to other individuals in such 
a space, among other things, models the level of relationships between them and involvement into the information exchange. The proximity of individuals to each other in this model suggests that there is a regular exchange of information between them, which establishes a social connection. The conflict in such a statement of the problem should be regarded as a variant of the interaction of individuals, or groups of individuals, as a result of which the distance (i.e., social distance $x_{i}-x_{j}$, where $x_{i}$ and $x_{j}$ are the coordinates in social and physical space, $i, j=[1, N]$, where $N$ is the number of individuals or consolidated groups of individuals) between them is growing rapidly.

\subsection{Mathematical Representation of the System}

The communicative field, as in [Petukhov, 2018], is represented by a diffusion equation with a divergent type of diffusion:

$$
\begin{aligned}
& \frac{\partial}{\partial t} h\left(x_{i}, t\right)= \\
& =\sum_{j=1}^{N} f\left(x_{i}, x_{j}\right) \vartheta\left(x_{i}, x_{j}\right) \bar{\delta}_{\left(k_{s}^{j}+k_{c}^{j}\right),\left(k_{s}^{i}+k_{c}^{i}\right)}+ \\
& +D\left(h\left(x_{i}, t\right)-h\left(x_{i}, t_{0}\right)\right)
\end{aligned}
$$

where $f\left(x_{i}, x_{j}\right)$ is a function that describes the interaction between individuals, which is modeled by the classical Gaussian distribution;

$$
\vartheta\left(x_{i}, x_{j}\right)=\frac{1}{\varepsilon \sqrt{\pi}} e^{\frac{-\left(x_{i}-x_{j}\right)^{2}}{\varepsilon^{2}}},
$$

Function $\vartheta\left(x_{i}, x_{j}\right)$ is introduced instead of the deltafunction to simplify the process of computer modeling; $\bar{\delta}_{\left(k_{s}^{j}+k_{c}^{j}\right),\left(k_{s}^{i}+k_{c}^{i}\right)}$ is the inverse Kronecker symbol; $D$ is the diffusion coefficient describing the propagation of the communicative field. The movement of an individual in space is described by the formula based on the Langevin equation:

$$
\frac{d x_{i}}{d t}=C(x) k_{s}^{i}\left(\sum_{j=1, j \neq i}^{N} \frac{\partial}{\partial x_{j}} h\left(x_{j}, t\right)\right)+\sqrt{2 D} \xi_{i}(t),
$$

$C(x)$ is the control function, which we set as:

$$
C(x)=-\frac{x_{i}}{\tau}
$$

where $\tau$ is the time of relaxation in the society. From a physical point of view, this function is a function of dissipation.

$k_{c}^{i}$ - coefficient of social activity of the $i^{t h}$ individual or a group of individuals,

$k_{s}^{i}$ - coefficient of the scientific and technological progress of the $i^{t h}$ individual or a group of individuals,

\section{$\xi_{i}(t)$ - stochastic force}

We believe that the distinctive parameters of the system can take on values:
In the general case, the following are chosen as the initial conditions for equations (1) and (2):

$$
\left.x_{i}\right|_{t=0}=x_{0 i}, \quad h\left(x_{i}, t=0\right)=h_{0 i}
$$

\subsection{An Approximate Solution of the System}

Let us consider a model of two interacting consolidated ethnic groups of individuals, presumably in a state of conflict. In this case, equations (1) and (2) produce four equations that fully describe the model of interaction of individuals:

$$
\left\{\begin{array}{l}
\frac{\partial h\left(x_{1}, t\right)}{\partial t}=D\left[h\left(x_{1}, t\right)-h\left(x_{1}, 0\right)\right]+ \\
+\alpha k_{c}^{2} k_{s}^{1} e^{-\frac{\psi^{2}+1}{\psi^{2}}\left(x_{1}-x_{2}\right)^{2}}, \\
\frac{\partial h\left(x_{2}, t\right)}{\partial t}=D\left[h\left(x_{2}, t\right)-h\left(x_{2}, 0\right)\right]+ \\
+\alpha k_{c}^{1} k_{s}^{2} e^{-\frac{\psi^{2}+1}{\psi^{2}}\left(x_{1}-x_{2}\right)^{2}}, \\
\frac{d x_{1}}{d t}=C\left(x_{1}\right)+k_{c}^{1} k_{s}^{1} \frac{\partial h\left(x_{2}, t\right)}{\partial x_{2}}+\sqrt{2 D} \xi_{1}(t), \\
\frac{d x_{2}}{d t}=C\left(x_{2}\right)+k_{c}^{2} k_{s}^{2} \frac{\partial h\left(x_{1}, t\right)}{\partial x_{1}}+\sqrt{2 D} \xi_{2}(t),
\end{array}\right.
$$

where:

$$
\psi=k_{c}^{1}+k_{s}^{1}+k_{c}^{2}+k_{s}^{2}, \alpha=\frac{1}{\psi \sqrt{\pi}} \bar{\delta}_{k_{c}^{1}+k_{s}^{1}, k_{c}^{2}+k_{s}^{2}} .
$$

Here, as in [Petukhov, 2018]: in order to obtain approximate analytic solutions of the system (3), we use the series expansion accurate to first-order quantities of smallness for $\Delta x=x_{i}-x_{o i}, \Delta t=t-t_{o}$ difference:

$$
\begin{gathered}
h\left(x_{i}, t\right)-h\left(x_{o i}, t_{o}\right) \approx \\
\left.\approx\left(\frac{\partial h}{\partial x_{i}}\right)\right|_{t=0, x_{i}=x_{0 i}} \Delta x+\left.\left(\frac{\partial h}{\partial t}\right)\right|_{t=0, x_{i}=x_{0 i}} \Delta t,
\end{gathered}
$$

Then, assuming that the following initial conditions are present:

$$
x_{o i}=0,
$$

$h\left(x_{o i}, t_{o}\right)=\left.\left(\frac{\partial h}{\partial x_{i}}\right)\right|_{t=0, x_{i}=x_{0 i}}=\left.\left(\frac{\partial h}{\partial t}\right)\right|_{t=0, x_{i}=x_{0 i}}=1$,

let us integrate the first two equations of the system (3), and then, using the obtained results and the two latter equations of the system (3), considering the continuity of the corresponding functions, transform the system. Further as [Petukhov, 2018]:

$$
\begin{gathered}
h\left(x_{i}, t\right)=D \int_{0}^{t} x_{i}(u) d u+D \frac{t^{2}}{2}+ \\
+\alpha k_{c}^{j} k_{s}^{i} \int_{0}^{t} e^{-\frac{\psi^{2}+1}{\psi^{2}}\left(x_{i}(u)-x_{j}(u)\right)^{2}} d u, \\
j=3-i .
\end{gathered}
$$

$$
0<k_{c}, k_{s}, D<1 \text {. }
$$


Using this expression, the last two equations of system (3) can be transformed, based on the continuity of all the functions, into the following form:

$$
\left\{\begin{array}{l}
\frac{d x_{1}}{d t}=k_{c}^{1} k_{s}^{1}\left[D t+2 \alpha k_{c}^{1} k_{s}^{2} \frac{\psi^{2}+1}{\psi^{2}} \times\right. \\
\left.\int_{0}^{t}\left(x_{1}(u)-x_{2}(u)\right) e^{-\frac{\psi^{2}+1}{\psi^{2}}\left(x_{1}(u)-x_{2}(u)\right)^{2}} d u\right]+ \\
+\sqrt{2 D} \xi_{1}(t) \\
\frac{d x_{2}}{d t}=k_{c}^{2} k_{s}^{2}\left[D t+2 \alpha k_{c}^{2} k_{s}^{1} \frac{\psi^{2}+1}{\psi^{2}} \times\right. \\
\left.\int_{0}^{t}\left(x_{2}(u)-x_{1}(u)\right) e^{-\frac{\psi^{2}+1}{\psi^{2}}\left(x_{1}(u)-x_{2}(u)\right)^{2}} d u\right]+ \\
+\sqrt{2 D} \xi_{2}(t) .
\end{array}\right.
$$

After time differentiation, the following forms of differential equations are obtained:

$$
\left\{\begin{array}{c}
\frac{d^{2} x_{1}}{d t^{2}}=k_{c}^{1} k_{s}^{1} D+\frac{2 \alpha\left(\psi^{2}+1\right) k_{c}^{1} k_{s}^{1} k_{c}^{1} k_{s}^{2}}{\psi^{2}} \times \\
\left(x_{1}-x_{2}\right) e^{-\frac{\psi^{2}+1}{\psi^{2}}\left(x_{1}-x_{2}\right)^{2}}+\sqrt{2 D} \frac{d \xi_{1}(t)}{d t} \\
\frac{d^{2} x_{2}}{d t^{2}}=k_{c}^{2} k_{s}^{2} D+\frac{2 \alpha\left(\psi^{2}+1\right) k_{c}^{2} k_{s}^{2} k_{c}^{2} k_{s}^{1}}{\psi^{2}} \times \\
\left(x_{2}-x_{1}\right) e^{-\frac{\psi^{2}+1}{\psi^{2}}\left(x_{1}-x_{2}\right)^{2}}+\sqrt{2 D} \frac{d \xi_{2}(t)}{d t}
\end{array}\right.
$$

Assuming that the stochastic forces for the two groups are the same $\xi_{1}(t)=\xi_{2}(t)$.

Then, by introducing new variables:

$$
\begin{aligned}
& y=x_{1}-x_{2}, \\
& A=D\left(k_{c}^{1} k_{s}^{1}-k_{c}^{2} k_{s}^{2}\right), \\
& B=2 \alpha \frac{\left(\psi^{2}+1\right)}{\psi^{2}}\left(k_{c}^{1} k_{s}^{1} k_{c}^{1} k_{s}^{2}+k_{c}^{2} k_{s}^{2} k_{c}^{2} k_{s}^{1}\right), \\
& C=\frac{\psi^{2}+1}{\psi^{2}},
\end{aligned}
$$

we obtain an equation that looks as follows:

$$
\begin{aligned}
& \frac{d^{2} y}{d t^{2}}=A-H \frac{d y}{d t}+B y e^{-C y^{2}}, \\
& B>0, C>0, H=\frac{1}{\tau},
\end{aligned}
$$

where $A, B, C$ depend on the parameters: $k_{s}^{i}, k_{c}^{i}, D$.

Let us write the equation (4) in the Cauchy form:

$$
\left\{\begin{array}{l}
\frac{d y}{d t}=z, \\
\frac{d z}{d t}=A-H z+B y e^{-C y^{2}} .
\end{array}\right.
$$

The system (5) can be viewed as a dynamic system that describes the process of interaction of two individuals or groups of individuals. This system is non-conservative, but finding its equilibrium states is reduced to solving the same system of equations as in the conservative case, see [Petukhov, 2018]:

$$
\left\{\begin{array}{l}
z=0 \\
y e^{-C y^{2}}=-\frac{A}{B} .
\end{array}\right.
$$

It was shown in [Petukhov, 2018] that the corresponding system has two equilibrium states: the saddle and the center. The general theory of dynamical systems states that the saddle is a rough equilibrium state, that is, its type does not change after a sufficiently small change in the system. While the center is a non-rough state of equilibrium, with small changes in the system, such a state of equilibrium shifts to a stable or unstable focus.

Taking into account what has been said about coarse and non-equilibrium equilibrium states, it is rather easy to construct a phase portrait for the system under consideration in the presence of dissipations of different types (as quadratic, where $x_{i}$ in the dissipation function is replaced by $x_{i}^{2}$, as well as with negative $\tau$ and positive). Following the above, the saddle state of equilibrium does not change its type, but the stable separatrix loop will break, while the center-type equilibrium state at $\tau>0$ will turn into an unstable focus (Fig. 1).

Figures 1-3 show phase portraits for the case of two equilibrium states under conditions

$$
0<-\frac{A}{B}<\sqrt{\frac{1}{2 C}} e^{-\frac{1}{2}}, A<0,
$$

for three different values of the parameter $\tau>0$ and $\tau<0$, where $\dot{y}=\frac{d y}{d t}$.

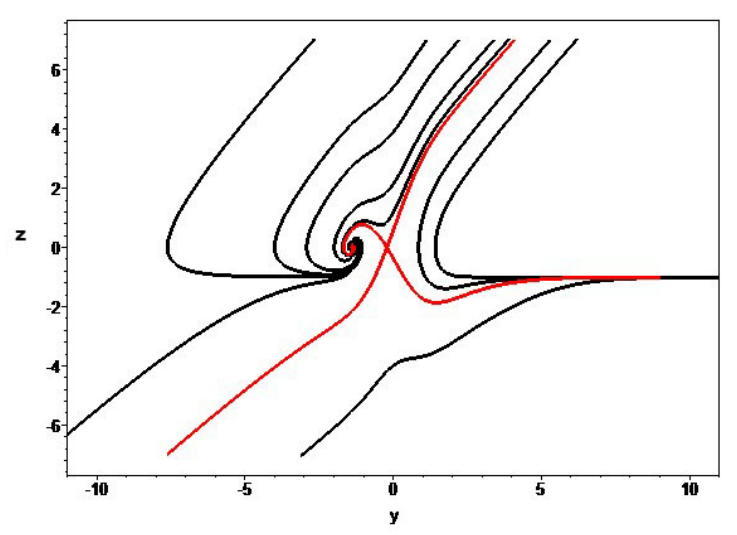

Figure 1. Linear additive. $\tau<0$. Unstable focus and saddle.

From the obtained phase portraits (Fig. 1 - Fig. 3), we see that we have two equilibrium states, a stable-unstable focus, depending on the dissipation function and the saddle. Red trajectories are separatrices of the saddle O_2.).

The transition from one equilibrium state to another a change in stability at the focus, is a classical threshold effect, at which a change in the region of attraction of the focus leads to completely different variants of the 


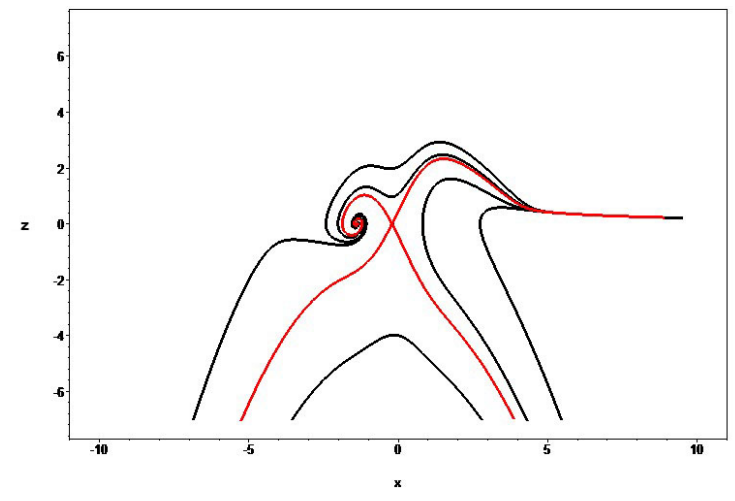

Figure 2. Quadratic additive. $\tau>0$. Unstable focus and saddle.

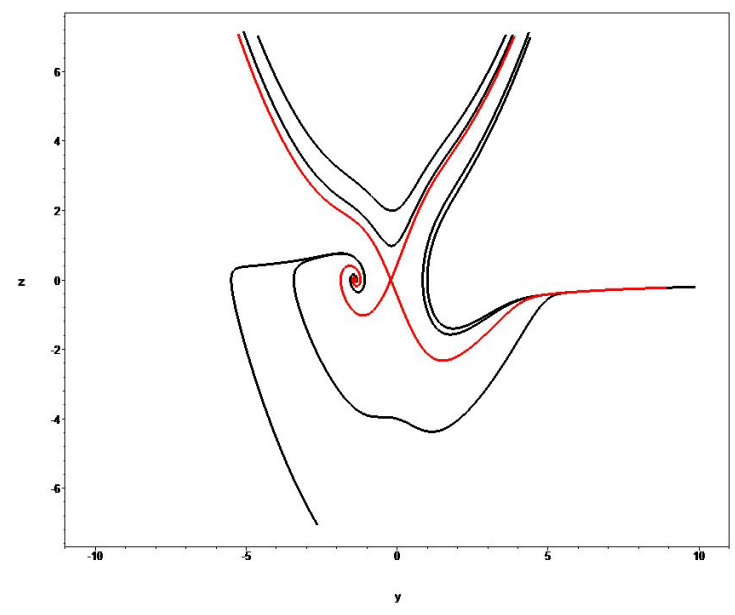

Figure 3. Quadratic additive. $\tau<0$. Stable focus and saddle.

evolution of the system. The separatrices diverge from an unstable focus, which means the absence of a region of stability [Petukhov, 2018] in terms of the emergence of social conflict.

In addition, by changing the parameters, it is not difficult to notice that the behavior of the phase trajectories also changes. Recent changes may consist in purely quantitative changes in the size and location of the trajectories, but they can lead to significant, qualitative changes in the structure of the phase portrait, that is, to bifurcation.

The threshold effects in this model can be caused by two factors: the bifurcation value of the parameters and the initial conditions of the problem (passing through the separatrices of the saddle from the asymptotic stability region to the instability region, where the trajectories converge).

\section{Conclusion}

This article proposed an approach to modeling social conflict and describing possible threshold effects. Given a formalized definition of one of the parameters leading to conflict in the social system. A mathematical model based on the Langevin equation is proposed, an analytical solution is proposed in the first approximation for a divergent type of diffusion. Specific boundary conditions were established, determined by the parameters of the social system and external influence, which created the basis for the emergence of social conflict and the appearance of threshold effects.

\section{Acknowledgements}

The study was supported by grants from the Russian Foundation for Basic Research No. 17-06-00640_a.

\section{References}

Abzalilov D.F. (2012). Mathematical modeling in sociology: Educational-methodical manual for sociologists. Kazan. CFU.

Castellano, C., Fortunato, S. \& Loreto, V. (2009). Statistical physics of social dynamics. Reviews of Modern Physics. 81, pp. 591-646.

Holyst J.A., Kasperski K., Schweitger F. (2000). Phase transitions in social impact models of opinion formation. Physica. A285, pp.199-210.

Malkov V.P. (2009). Mathematical modeling of historical dynamics: approaches and models. $\mathrm{M}$.

Plotnitskiy Yu. M. (2001). Models of Social Processes: Textbook for Higher Education Institutions. M. Logos.

Petukhov A.Y. (2015). Modelling of social conflict: Socio-energy approach. Global Journal of Pure and Applied Mathematics. 11(6), pp. 4197-4206.

Petukhov A.Y., Malkhanov A.O., Sandalov V.M., Petukhov Y.V. (2018). Modeling conflict in a social system using diffusion equations. Simulation. April 16, pp. 1-9. DOI: $10.1177 / 0037549718761573$

Smith, L. M., Lerman, K., Garcia-Cardona, C., Percus, A. G. \& Ghosh, R. (2013). Spectral clustering with epidemic diffusion. Physical Review E. 88, pp. 042813.

Traud, A. L., Kelsic, E. D., Mucha, P. J. \& Porter, M. A. (2011). Comparing community structure to characteristics in online collegiate social networks. SIAM Review. 53, pp. 526-543. 